УДК 517.55

\title{
An Integral Formula for the Number of Lattice Points in a Domain
}

\author{
Lev Aizenberg* \\ Department of Mathematics \\ Bar-Ilan University \\ 52900 Ramat-Gan \\ Israel \\ Nikolai Tarkhanov ${ }^{\dagger}$ \\ Institute of Mathematics \\ University of Potsdam \\ Am Neuen Palais, 10, Potsdam, 14469
}

Germany

Received 06.02.2015, received in revised form 06.03.2015, accepted 14.04.2015

Using the multidimensional logarithmic residue we show a simple formula for the difference between the number of integer points in a bounded domain of $\mathbb{R}^{n}$ and the volume of this domain. The difference proves to be the integral of an explicit differential form over the boundary of the domain.

Keywords: logarithmic residue, lattice point.

\section{Introduction}

Classical function theory is of great importance in number theory, let alone the analytical extension of the Riemann zeta function and prime number theorem, see $[6,8,9]$, etc.

This work was intended as an attempt at applying the theory of functions of several complex variables to classical problems of number theory. To wit, we apply the multidimensional logarithmic residue which is an efficient numerical tool of algebraic geometry, see [1].

Let $\mathcal{Z}$ be a bounded domain with piecewise smooth boundary in the space $\mathbb{C}^{n}$ of $n$ complex variables $z=\left(z_{1}, \ldots, z_{n}\right)$. Consider a holomorphic mapping $w=f(z)$ of the closed domain $\overline{\mathcal{Z}}$ into $\mathbb{C}^{n}$ which has no zeros at the boundary of $\mathcal{Z}$. Then $f$ has only isolated zeros in $\mathcal{Z}$ and the number of zeros counted with their multiplicity is given by the logarithmic residue formula

$$
N(f, \mathcal{Z})=\int_{\partial \mathcal{Z}} \frac{(n-1) !}{(2 \pi \imath)^{n}} \sum_{j=1}^{n}(-1)^{j-1} \frac{\bar{f}_{j}}{|f|^{2 n}} d \bar{f}[j] \wedge d f
$$

(see $[1, \S 2])$, where $|f|^{2}=\left|f_{1}\right|^{2}+\ldots+\left|f_{n}\right|^{2}, f_{j}$ being the $j$ th component of $f$, by $d f=d f_{1} \wedge \ldots \wedge d f_{n}$ is meant the exterior product of the differentials $d f_{1}, \ldots, d f_{n}$, and $d \bar{f}[j]$ stands for the exterior product of the differentials $d \bar{f}_{1}, \ldots, d \bar{f}_{n}$ after each other, the differential $d \bar{f}_{j}$ being omitted. The domain $\mathcal{Z}$ is oriented in such a way that

$$
\int_{\mathcal{Z}} \frac{1}{(2 \imath)^{n}} d \bar{z} \wedge d z>0
$$

We apply formula (1) to get an equality for the difference between the number of lattice points in the domain $\mathcal{Z}$ and its volume. A number of classical problems of number theory, e.g. the

\footnotetext{
*aizenbrg@gmail.com

†tarkhanov@math.uni-potsdam.de

(c) Siberian Federal University. All rights reserved
} 
problem on the number of lattice points in a ball [10], the problems on Dirichlet divisors [4], etc. reduce to evaluating asymptotics of the difference. It is worth pointing out that this asymptotics can not be found by standard methods, such as the Laplace method, stationary phase method, or saddle point method.

The theory of lattice points in large regions has attracted the interest of many mathematicians for more than eleven decades. The monograph [5] presents a broad survey of the main problems and results in lattice point theory.

\section{The integral formula}

As usual, we write $\mathbb{R}^{n}, n \geqslant 1$, for the $n$-dimensional real Euclidean space of variables $x=$ $\left(x_{1}, \ldots, x_{n}\right)$ with $x_{j} \in \mathbb{R}$. Suppose $\mathcal{X}$ is a bounded domain in $\mathbb{R}^{n}$ whose boundary is piecewise smooth and does not contain any point with integer coordinates. Denote by $N(\mathcal{X})$ the number of integer points in $\mathcal{X}$ and by $V(\mathcal{X})$ its volume.

Theorem 1. If the boundary $\partial \mathcal{X}$ does not contain lattice points then the difference $N(\mathcal{X})-V(\mathcal{X})$ can be written in the form

$$
N(\mathcal{X})-V(\mathcal{X})=\int_{0}^{\infty} \ldots \int_{0}^{\infty} d t \int_{\partial \mathcal{X}} \frac{2^{n-2}(n-1) !}{\pi} \frac{\sum_{j=1}^{n} t[j] \sin \left(2 \pi x_{j}\right) \nu_{j}}{\left(\sum_{j=1}^{n}\left(t_{j}^{2}-2 t_{j} \cos \left(2 \pi x_{j}\right)\right)+n\right)^{n}} d s
$$

where $d t=d t_{1} \wedge \ldots \wedge d t_{n}, t[j]=t_{1} \ldots t_{j-1} t_{j+1} \ldots t_{n}$, ds is the surface measure of $\partial \mathcal{X}$ and $\nu(x)=\left(\nu_{1}(x), \ldots, \nu_{n}(x)\right)$ is the unit outward normal vector of the boundary at $x \in \partial \mathcal{X}$.

Proof. Consider the domain $\mathcal{Z}=\mathcal{X} \times \mathcal{Y}$ in $\mathbb{C}^{n}$, where $\mathcal{Y}$ is a bounded domain with piecewise smooth boundary in the space $\mathbb{R}^{n}$ of variables $y=\left(y_{1}, \ldots, y_{n}\right)$. We assume that $0 \in \mathcal{Y}$. The points $z=\left(z_{1}, \ldots, z_{n}\right)$ of $\mathcal{Z}$ have the form $z_{j}=x_{j}+\imath y_{j}$, for $j=1, \ldots, n$. As holomorphic mapping $f: \mathcal{Z} \rightarrow \mathbb{C}^{n}$ vanishing solely at the entire points of $\mathcal{X} \times\{0\}$, we take

$$
\begin{aligned}
f_{1}(z) & =e^{2 \pi \imath z_{1}}-1, \\
& \cdots \\
f_{n}(z) & =e^{2 \pi \imath z_{n}}-1,
\end{aligned}
$$

each zero being simple.

By formula (1), we get

$$
N(f, \mathcal{Z})=\int_{\partial \mathcal{Z}} \frac{(n-1) !}{(2 \pi \imath)^{n}} \frac{\sum_{j=1}^{n}(-1)^{j-1}\left(e^{-2 \pi \imath \bar{z}_{j}}-1\right) d e^{-2 \pi \imath \bar{z}}[j] \wedge d e^{2 \pi \imath z}}{\left(\left|e^{2 \pi \imath z_{1}}-1\right|^{2}+\ldots+\left|e^{2 \pi \imath z_{n}}-1\right|^{2}\right)^{n}},
$$

where

$$
\begin{aligned}
d e^{-2 \pi \imath \bar{z}}[j] & =d e^{-2 \pi \imath \bar{z}_{1}} \wedge \ldots \wedge d e^{-2 \pi \imath \bar{z}_{j-1}} \wedge d e^{-2 \pi \imath \bar{z}_{j+1}} \wedge \ldots \wedge d e^{-2 \pi \imath \bar{z}_{n}}, \\
d e^{2 \pi \imath z} & =d e^{2 \pi \imath z_{1}} \wedge \ldots \wedge d e^{2 \pi \imath z_{n}} .
\end{aligned}
$$

The right-hand side is easily reduced to

$$
\begin{gathered}
\int_{\partial \mathcal{Z}}(-2 \pi \imath)^{n-1}(n-1) ! \frac{\sum_{j=1}^{n}(-1)^{j-1}\left(e^{-2 \pi \imath \sum_{k=1}^{n} \bar{z}_{k}}-e^{-2 \pi \imath \sum_{k \neq j} \bar{z}_{k}}\right) e^{2 \pi \imath \sum_{k=1}^{n} z_{k}} d \bar{z}[j] \wedge d z}{\left(\left|e^{2 \pi \imath z_{1}}-1\right|^{2}+\ldots+\left|e^{2 \pi \imath z_{n}}-1\right|^{2}\right)^{n}} \\
-135-
\end{gathered}
$$


A trivial verification shows that

$$
d \bar{z}[j] \wedge d z=(2 \imath)^{n-1}\left((-1)^{n-1} d x \wedge d y[j]+\imath d x[j] \wedge d y\right)
$$

for all $j=1, \ldots, n$. Using (4) one separates the real and imaginary parts of (3), these are

$$
\begin{array}{r}
\int_{\partial \mathcal{Z}}(4 \pi)^{n-1}(n-1) ! \frac{\sum_{j=1}^{n}(-1)^{n+j}\left(e^{-4 \pi \sum_{k=1}^{n} y_{k}}-e^{-4 \pi \sum_{k \neq j} y_{k}-2 \pi y_{j}} \cos \left(2 \pi x_{j}\right)\right) d x \wedge d y[j]}{\left(\left|e^{2 \pi \imath z_{1}}-1\right|^{2}+\ldots+\left|e^{2 \pi \imath z_{n}}-1\right|^{2}\right)^{n}}+ \\
+\int_{\partial \mathcal{Z}}(4 \pi)^{n-1}(n-1) ! \frac{\sum_{j=1}^{n}(-1)^{j-1} e^{-4 \pi \sum_{k \neq j} y_{k}-2 \pi y_{j}} \sin \left(2 \pi x_{j}\right) d x[j] \wedge d y}{\left(\left|e^{2 \pi \imath z_{1}}-1\right|^{2}+\ldots+\left|e^{2 \pi \imath z_{n}}-1\right|^{2}\right)^{n}}
\end{array}
$$

and

$$
\begin{gathered}
\int_{\partial \mathcal{Z}}(4 \pi)^{n-1}(n-1) ! \frac{\sum_{j=1}^{n}(-1)^{j-1}\left(e^{-4 \pi \sum_{k=1}^{n} y_{k}}-e^{-4 \pi \sum_{k \neq j} y_{k}-2 \pi y_{j}} \cos \left(2 \pi x_{j}\right)\right) d x[j] \wedge d y}{\left(\left|e^{2 \pi \imath z_{1}}-1\right|^{2}+\ldots+\left|e^{2 \pi \imath z_{n}}-1\right|^{2}\right)^{n}}+ \\
+\int_{\partial \mathcal{Z}}(4 \pi)^{n-1}(n-1) ! \frac{\sum_{j=1}^{n}(-1)^{n+j-1} e^{-4 \pi \sum_{k \neq j} y_{k}-2 \pi y_{j}} \sin \left(2 \pi x_{j}\right) d x \wedge d y[j]}{\left(\left|e^{2 \pi \imath z_{1}}-1\right|^{2}+\ldots+\left|e^{2 \pi \imath z_{n}}-1\right|^{2}\right)^{n}}
\end{gathered}
$$

respectively.

The number $N(f, \mathcal{Z})$ is real, hence it suffices to consider the mere real part (5) of formula (1). Moreover, we make the change of variables

$$
\begin{aligned}
t_{1} & =e^{-2 \pi y_{1}}, \\
& \cdots \\
t_{n} & =e^{-2 \pi y_{n}},
\end{aligned}
$$

obtaining

$$
\begin{aligned}
N(f, \mathcal{Z}) & =\int_{\partial \mathcal{Z}^{\prime}} 2^{n-1}(n-1) ! \frac{\sum_{j=1}^{n}(-1)^{j-1} t_{1} \ldots t_{n}\left(t_{j}-\cos \left(2 \pi x_{j}\right)\right) d t[j] \wedge d x}{\left(\sum_{j=1}^{n}\left(t_{j}^{2}-2 t_{j} \cos \left(2 \pi x_{j}\right)\right)+n\right)^{n}}+ \\
& +\int_{\partial \mathcal{Z}^{\prime}} \frac{2^{n-2}(n-1) !}{\pi} \frac{\sum_{j=1}^{n}(-1)^{n+j-1} t[j] \sin \left(2 \pi x_{j}\right) d t \wedge d x[j]}{\left(\sum_{j=1}^{n}\left(t_{j}^{2}-2 t_{j} \cos \left(2 \pi x_{j}\right)\right)+n\right)^{n}}=I_{1}+I_{2},
\end{aligned}
$$

where $\mathcal{Z}^{\prime}$ is the image of the domain $\mathcal{Z}$ under the change of variables $t_{j}=e^{-2 \pi y_{j}}$, for $j=1, \ldots, n$.

This change involves the mere variables $y$ whence $\mathcal{Z}^{\prime}=\mathcal{X} \times \mathcal{T}$, where $\mathcal{T}$ is the image of $\mathcal{Y}$ by $t_{j}=e^{-2 \pi y_{j}}$ with $j=1, \ldots, n$. Since $\mathcal{Y}$ contains the origin, the $n$-tuple with coordinates 1 belongs to $\mathcal{T}$. We now give the domain $\mathcal{T}$ the following concrete form

$$
\mathcal{T}=\left\{t \in \mathbb{R}^{n}: r^{2}<|t|^{2}<R^{2}\right\} \cap\left\{t \in \mathbb{R}^{n}: t_{1}, \ldots, t_{n}>\varepsilon\right\}
$$


where $r<\sqrt{n}, R>\sqrt{n}$ and $\varepsilon>0$ is small enough. The boundary $\partial \mathcal{T}$ consists of a piece $S_{r}$ of the $(n-1)$-dimensional sphere $\left\{t \in \mathbb{R}^{n}:|t|=r\right\}$, a piece $S_{R}$ of the $(n-1)$-dimensional sphere $\left\{t \in \mathbb{R}^{n}:|t|=R\right\}$, and pieces $H_{j}$ of hypersurfaces $t_{j}=\varepsilon$ parallel to the coordinates hyperplanes $t_{j}=0$. According to this structure of the boundary of $\mathcal{T}$ we represent the integral $I_{1}$ as the sum of integrals $I_{1, S_{r}}, I_{1, S_{R}}$ and $I_{1, H_{j}}$ with $j=1, \ldots, n$.

Let the piece $H_{1}$ tend to the hyperplane $\left\{t_{1}=0\right\}$. At this hyperplane we obviously get

$$
\sum_{j=1}^{n}\left(t_{j}^{2}-2 t_{j} \cos \left(2 \pi x_{j}\right)\right)+n=\sum_{j=2}^{n}\left(t_{j}-\cos \left(2 \pi x_{j}\right)\right)^{2}+\sin ^{2}\left(2 \pi x_{j}\right)+1 \geqslant 1 .
$$

Therefore, the integral $I_{1, H_{1}}$ tends to zero as $H_{1}$ tends to the hyperplane $\left\{t_{1}=0\right\}$. Analogously, $I_{1, H_{j}}$ tends to zero as $H_{j}$ tends to the hyperplane $\left\{t_{j}=0\right\}$, for each $j=2, \ldots, n$.

It remains to consider the limits of the integrals $I_{1, S_{r}}$ and $I_{1, S_{R}}$, when $r \rightarrow 0$ and $R \rightarrow \infty$. Let $\mathbb{S}_{\geqslant 0}^{n-1}$ be the part of the unit sphere with centre at the origin which lies in the cube $0 \leqslant t_{j} \leqslant 1$, $j=1, \ldots, n$. We endow $\mathbb{S}_{\geqslant 0}^{n-1}$ with the usual orientation, then $S_{r}=-r \mathbb{S}_{\geqslant 0}^{n-1}$ and $S_{R}=R \mathbb{S}_{\geqslant 0}^{n-1}$. (When we tended $H_{j}$ to the hyperplane $\left\{t_{j}=0\right\}$ for all $j=1, \ldots, n$, then $S_{r}$ and $S_{R}$ became one $2^{n}$-th spheres.) Hence it follows readily that

$$
I_{1, S_{r}}=-\int_{\mathcal{X}} d x \int_{\mathbb{S}_{\geqslant 0}^{n-1}} 2^{n-1}(n-1) ! \frac{\sum_{j=1}^{n}(-1)^{j-1} r^{2 n-1} t_{1} \ldots t_{n}\left(r t_{j}-\cos \left(2 \pi x_{j}\right)\right) d t[j]}{\left(r^{2}-2 \sum_{j=1}^{n} r t_{j} \cos \left(2 \pi x_{j}\right)+n\right)^{n}} \rightarrow 0
$$

as $r \rightarrow 0$.

On the other hand, we get

$$
\begin{aligned}
I_{1, S_{r}} & =\int_{\mathcal{X}} d x \int_{\mathbb{S}_{\geqslant 0}^{n-1}} 2^{n-1}(n-1) ! \frac{\sum_{j=1}^{n}(-1)^{j-1} R^{2 n-1} t_{1} \ldots t_{n}\left(R t_{j}-\cos \left(2 \pi x_{j}\right)\right) d t[j]}{\left(R^{2}-2 \sum_{j=1}^{n} R t_{j} \cos \left(2 \pi x_{j}\right)+n\right)^{n}} \rightarrow \\
& \rightarrow \int_{\mathcal{X}} d x \int_{\mathbb{S}_{\geqslant 0}^{n-1}} 2^{n-1}(n-1) ! \sum_{j=1}^{n}(-1)^{j-1} t_{1} \ldots t_{n} t_{j} d t[j],
\end{aligned}
$$

as $R \rightarrow \infty$. The last integral just amounts to $V(\mathcal{X})$, for

$$
\int_{\mathbb{S}_{\geqslant 0}^{n-1}} 2^{n-1}(n-1) ! \sum_{j=1}^{n}(-1)^{j-1} t_{1} \ldots t_{n} t_{j} d t[j]=\int_{\mathbb{S}_{\geqslant 0}^{n-1}} 2^{n-1}(n-1) ! t_{1} \ldots t_{n} d s=1 .
$$

Thus, if the domain $\mathcal{T}$ expands to the nonnegative one $2^{n}$-th space as above, the integral $I_{1}$ tends to $V(\mathcal{X})$. And the integral $I_{2}$ converges to the integral on the right-hand side of formula $(2)$, for $\partial \mathcal{Z}^{\prime}=(\partial \mathcal{X} \times \mathcal{T}) \cup\left(\mathcal{X} \times \partial \mathcal{T}\right.$ and $(-1)^{j-1} d x[j]=\nu_{j} d s$ for all $j=1, \ldots, n$, as desired.

For the most practical cases $n=2$ and $n=3$ Theorem 1 was first proved in [2].

\section{The one-dimensional case}

In this section we clarify the structure of formula (2) by directly computing the integral on the right-hand side of this formula in the case $n=1$. Let $\mathcal{X}=(a, b)$, where $m<a<m+1$ and 
$M<b<M+1, m$ and $M$ being integer numbers satisfying $m<M$. Then

$$
\begin{aligned}
I & =\int_{0}^{\infty} d t \int_{\partial \mathcal{X}} \frac{1}{2 \pi} \frac{\sin 2 \pi x}{(t-\cos 2 \pi x)^{2}+(\sin 2 \pi x)^{2}}= \\
& =\int_{0}^{\infty} \frac{1}{2 \pi}\left(\frac{\sin 2 \pi b}{(t-\cos 2 \pi b)^{2}+(\sin 2 \pi b)^{2}}-\frac{\sin 2 \pi a}{(t-\cos 2 \pi a)^{2}+(\sin 2 \pi a)^{2}}\right) d t .
\end{aligned}
$$

Substituting $s=t-\cos 2 \pi b$ and $s=t-\cos 2 \pi a$ into the first and second terms on the right-hand side, respectively, we get

$$
\begin{aligned}
I & =\int_{-\cos 2 \pi b}^{\infty} \frac{1}{2 \pi} \frac{\sin 2 \pi b}{s^{2}+(\sin 2 \pi b)^{2}} d s-\int_{-\cos 2 \pi a}^{\infty} \frac{1}{2 \pi} \frac{\sin 2 \pi a}{s^{2}+(\sin 2 \pi a)^{2}} d s= \\
& =\left.\frac{1}{2 \pi} \arctan \frac{s}{\sin 2 \pi b}\right|_{-\cos 2 \pi b} ^{\infty}-\frac{1}{2 \pi} \arctan \frac{s}{\sin 2 \pi a} \Gamma_{-\cos 2 \pi a}^{\infty} .
\end{aligned}
$$

To be specific, we consider the case

$$
\begin{aligned}
m+1 / 2 & <a<m+1, \\
M & <b<M+1 / 2,
\end{aligned}
$$

then $\sin 2 \pi a<0$ and $\sin 2 \pi b>0$. Hence it follows that

$$
\begin{aligned}
I & =\frac{1}{2 \pi}\left(\frac{\pi}{2}-\arctan \left(-\frac{\cos 2 \pi b}{\sin 2 \pi b}\right)-\left(-\frac{\pi}{2}\right)+\arctan \left(-\frac{\cos 2 \pi a}{\sin 2 \pi a}\right)\right)= \\
& =\frac{1}{2 \pi}(\pi+\arctan \cot 2 \pi b-\arctan \cot 2 \pi a) .
\end{aligned}
$$

Finally, on using the equality $\arctan x=\pi / 2-\operatorname{arccot} x$ we deduce

$I=\frac{1}{2 \pi}(\pi-\arctan \cot (2 \pi b-2 \pi M)+\arctan \cot (2 \pi a-2 \pi(m+1 / 2))=(M-m)-(b-a)$,

which just amounts to $N(\mathcal{X})-V(\mathcal{X})$, as desired.

\section{Some comments}

It is easy to see that the integrations over $t \in[0, \infty)^{n}$ and $x \in \partial \mathcal{X}$ in formula (2) can be exchanged. In this way we get

$$
N(\mathcal{X})-V(\mathcal{X})=\int_{\partial \mathcal{X}} \sum_{j=1}^{n}(-1)^{j-1} F_{j}(x) \sin 2 \pi x_{j} d x[j],
$$

where

$$
F_{j}(x)=\frac{2^{n-2}(n-1) !}{\pi} \int_{0}^{\infty} \ldots \int_{0}^{\infty} \frac{t[j]}{\left(\sum_{k=1}^{n}\left(t_{k}-\cos 2 \pi x_{k}\right)^{2}+\sum_{k=1}^{n}\left(\sin 2 \pi x_{k}\right)^{2}\right)^{n}} d t
$$

are functions of $\cos 2 \pi x_{j}$ and $\sin 2 \pi x_{j}$, for $j=1, \ldots, n$. The differential form under the integral over $\partial \mathcal{X}$ on the right-hand side of (7) is smooth away from the lattice of half-integer points in $\mathbb{R}^{n}$. As is seen from Section 2, the differential form is not closed outside this lattice. The coefficients $F_{j}$ bear certain symmetry in variables $x_{1}, \ldots, x_{n}$, perhaps, it suffices to compute only one of these coefficients in order to determine the others. Moreover, $F_{j}$ can be computed in a closed 
form, however, the expressions are cumbersome, cf. formula (3) in [3]. It is possible that formula (7) can be applied to construct asymptotics of the difference $N(\mathcal{X})-V(\mathcal{X})$ as $R \rightarrow \infty$, where $\mathcal{X}$ is the ball of radius $R$ with centre at 0 or, more generally, an ellipsoid

$$
\left(\frac{x_{1}}{a_{1}}\right)^{2}+\ldots+\left(\frac{x_{n}}{a_{n}}\right)^{2}<R^{2}
$$

or another expanding domain, cf. [5,7]. But we will not develop this point here.

This research was supported by the German Research Society (DFG), grant TA 289/4-2. The first author wishes to express his gratitude to Professor Sinai Robins who payed attention to the paper [2], invited the author to attend Singapore for two weeks and thus recommenced his interest to the topic.

\section{References}

[1] L.A.Aizenberg, A.P.Yuzhakov, Integral Representations and Residues in Multidimensional Complex Analysis, AMS, RI, 1983.

[2] L.Aizenberg, Application of multidimensional logarithmic residue for representation in the form of the integral of the difference between the number of integer points in a domain and its volume, Dokl. Akad. Nauk SSSR, 270(1983), no. 3, 521-523 (in Russian).

[3] L.Aizenberg, Application of multidimensional logarithmic residue in number theory, An integral formula for the difference between the number of lattice points in a domain and its volume, Annales Polonici Mathematici, 46(1985), 395-401.

[4] K.S.Chandrasekharan, Introduction to Analytic Number Theory, Springer, New York, 1968.

[5] F.Fricker, Introduction to Lattice Point Theory, Textbooks and Monographs in the Exact Sciences, Mathematical Series, 73, Birkhäuser Verlag, Basel-Boston, Mass., 1982.

[6] Th.W.Gamelin, Complex Analysis, Springer, New York et al., 2001.

[7] R.K.Guy, Unsolved Problems in Number Theory, Springer, New York et al., 1981.

[8] D.J.Newman, Analytic Number Theory, Springer, New York et al., 1998.

[9] E.C.Titchmarsh, The Theory of the Riemann Zeta-Function, Oxford University Press, London, 1951.

[10] I.M.Vinogradov, Particular Versions of Trigonometric Sum Method, Nauka, Moscow, 1976 (in Russian).

\section{Интегральная формула для числа целых точек в области}

\section{Лев Айзенберг \\ Николай Тарханов}

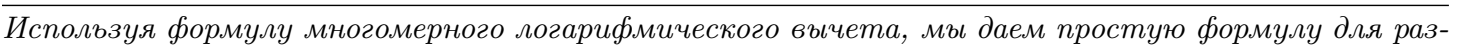
ности между числом целых точек в ограниченной области из $\mathbb{R}^{n}$ и обвемом этой области. Эта разность дается интегралом от дифференциальной формы, задаваемой точным выражением, по границе этой области.
}

Ключевые слова: логарифмический вычет, целая точка. 